\title{
The Consistency of Combat Exposure Reporting and Course of PTSD in Vietnam War Veterans
}

\author{
K. C. Koenen \\ Departments of Society, Human Development, and Health and Epidemiology, Harvard School \\ of Public Health, Boston, MA and Department of Psychiatry, Boston University School of \\ Medicine, Boston, $M A$ \\ S. D. Stellman \\ Department of Epidemiology, Mailman School of Public Health, Columbia University, \\ New York, NY \\ B. P. Dohrenwend \\ Department of Psychiatry, Columbia University and New York State Psychiatric Institute and \\ Department of Epidemiology, Mailman School of Public Health, Columbia University, \\ New York, NY \\ J. F. Sommer, Jr. \\ American Legion, Washington. D.C. \\ J. M. Stellman \\ Department of Health Policy and Management, Mailman School of Public Health, \\ Columbia University, New York, NY
}

\begin{abstract}
Self-reports of traumatic events are often used in clinical and epidemiologic studies. Nevertheless, research suggests combat exposure reports may be biased by posttraumatic stress disorder (PTSD) symptom severity, leading to an inflated dose-response relation between combat exposure and PTSD. The authors examined the consistency in combat exposure reports and their relation to PTSD symptoms in Vietnam Veteran American Legionnaires who responded to two mailed surveys (1984, 1998; $N=1,462)$. Combat exposure reports were highly reliable (test-retest correlation $=0.87$ ). However, changes in exposure reporting were related to changes in PTSD symptoms, specifically reexperiencing symptoms. The effect size of the dose-response relation attributable to changes in reporting was smaller for continuous than categorical measures. Findings are discussed in relation to recent controversies over veterans' combat exposure reports.
\end{abstract}

Posttraumatic stress disorder (PTSD) as a diagnosis originally developed out of research on the effects of combat exposure on Vietnam veterans. The strong doseresponse relation between severity of combat exposure and PTSD symptoms has been well-documented (Kulka et al., 1990; Snow, Stellman, Stellman, \& Sommer, 1988). Recent research on Gulf War and Somali peacekeeping mission veterans, however, has found that war-zone exposure reporting changed over time, with changes correlated with changes in PTSD symptoms (King \& King,

Correspondence concerning this article should be addressed to: J. M. Stellman, Department of Health Policy and Management, Mailman School of Public Health, Columbia University, New York, NY 10032. E-mail: jms13@columbia.edu. 
1991; Roemer, Litz, Orsillo, Ehlich, \& Friedman, 1998; Southwick, Morgan, Nicolaou, \& Charney, 1997). These findings have raised questions about the dose-response relation between combat exposure reports and PTSD in Vietnam veterans. That is, the observed dose-response relation between Vietnam combat exposure and PTSD may be subject, at least in part, to recall bias. This study examines the consistency of combat exposure reports and their relation to changes in PTSD symptoms in a large community cohort of Vietnam theater veterans followed over a 14-year period.

Measurement of war-zone exposure relies on respondents' recall of their experiences, usually in response to closed, self-report questions about the presence or absence of various types of combat exposure, often in the form of checklists or a large list of items with fixed response categories (King et al., 2000; Roemer et al., 1998; Southwick et al., 1997). The reliability and validity of such self-report measures has long been a research issue (King $\&$ King, 1991). A large body of literature has documented the phenomenon of mood-dependent memory whereby an individual's recall of experiences is affected by current psychological state (Cohen, Towbes, \& Flocco, 1988). Because combat exposure checklists often contain subjective items that may be reinterpreted according to the respondent's current psychological state, extension of the concept of current mood based bias to PTSD suggests that the recall of extreme combat exposure events could be biased by current PTSD symptomatology. Specifically, the presence or absence of PTSD could lead to differential reporting of negative past events. Reexperiencing the trauma through intrusive memories is part of the PTSD syndrome; therefore, such memories may be more accessible to individuals with more PTSD symptoms.

We investigated the reliability of combat exposure reporting and its relation to PTSD symptoms in Vietnam theater veterans who participated in a mailed health survey in 1984 and 1998. Identical measures of combat exposure and PTSD were used in both surveys, allowing us to examine changes in combat exposure reporting over 14 years, a period much longer than that examined in other studies. Furthermore, although most studies documenting the dose-response relation between combat exposure reports and PTSD have focused on Vietnam veterans, differential recall has not been studied in this population. One report on reliability of combat exposure reporting found a 6-month test-retest reliability of 0.84 , but did not examine differential recall as a function of PTSD symptoms (Janes, Goldberg, Eisen, True, \& Henderson, 1992). Vietnam veterans' wartime experiences were very different from those of the Gulf War or Somali peacekeeping mission, two groups previously reported on with regard to recall bias. The Vietnam War was the longest war in American history (1961-1975), involved many more troops, produced a much higher number of casualties, and received much less public support (Kulka et al., 1990). In addition, the Vietnam War has been depicted more frequently in the popular media than have these other conflicts.

In this study, we posed the following three questions: What is the reliability of combat exposure reported 14 years apart? Are changes in combat exposure reports associated with changes in PTSD symptoms or presumptive PTSD diagnosis? What is the degree to which changes in combat exposure reports affect the dose-response relation with PTSD symptoms/diagnosis?

\section{METHOD}

\section{Participants}

The cohort was originally surveyed in 1984 for a study of the health and well-being of American Legion members (Snow et al., 1988; J. M. Stellman, S. D. Stellman, \& Sommer, 1988a, 1988b; S. D. Stellman, J. M. Stellman, \& Sommer, 1988a, 1988b). The American Legion is the largest veterans' organization in the country, with 2.8 million members. Between 850,000 and 900,000 served during the Vietnam War era, of whom approximately $40 \%$ served in the Vietnam theatre of operations. A random sample of 12,000 male members of the American Legion who in October, 1983, belonged to any American Legion Post in Colorado, Indiana, Maryland, Minnesota, Ohio, or Pennsylvania, was drawn from post rosters. Details of the sampling method are described elsewhere (S. D. Stellman et al., 1988a). 
The first wave of the survey (Time 1) achieved a response rate of $56.3 \%$, with 2,860 (42\%) having served in the Vietnam theatre. A small number of veterans were found to be ineligible after returning their questionnaires. In 1998, we undertook a methodological study of exposure to herbicides and sought to reconstruct the original cohort to develop an approach for controlling for psychosocial health effects in our herbicide health studies. We made extensive efforts to locate the 1984 random sample, especially those who had dropped their Legion membership in the interim or who may have died. These efforts included use of private tracing firms, online Yellow Pages, commercial name and address listings, accession of military record data from the National Personnel Records Center (U.S. National Archives and Records Administration, St. Louis, MO), and networking within the American Legion. Through these efforts, 8,088 eligible veterans were located.

\section{Time 2 Surveu Procedures}

In 1998, a second questionnaire, nearly identical to the 1984 version, was mailed to 8,088 cohort members (Time 2) who included both veterans who responded to the Time 1 survey and those who did not. Nonrespondents to the Time 2 survey were followed-up with several repeat mailings and additional inquiries. We received responses from 4,490 men, for a response rate of $55.5 \%$. Of the 4,490, 3,403 responded at both Time 1 and Time 2. The cohort studied in the present analysis consists of 1,462 veterans who (a) returned surveys at both Time 1 and Time 2, (b) served in the Vietnam theater, (c) had reports of combat exposure that could be evaluated, and (d) could be assessed as having PTSD at both time periods.

The Institutional Review Board of the Columbia College of Physicians and Surgeons approved the study protocol, survey instruments, and explanatory cover letters for 1984 and 1998. The cover letter that accompanied the survey explained the purpose of the study and gave assurance that participation was voluntary, that responses would be maintained with strict confidence, and that the subject could withdraw at any time without prejudice. The survey development was described in more detail in previous reports (Koenen, Stellman, Stellman, \& Somers, 2003; Snow et al., 1988; J. M. Stellman et al., 1988a, 1988b; S. D. Stellman et al., 1988a, 1988b). At both times, the questionnaires included items on demographic information and a detailed military service history (S. D. Stellman et al., 1988a).

Vietnam combat exposure severity was assessed with an eight-question Likert-type scale with five possible responses that has previously been validated in studies of Vietnam veterans (Figley, 1978; Frye \& Stockton, 1982; S. D. Stellman et al., 1988). The questions, covering the extent of enemy fire and life-threatening situational exposures, were summed with a total score ranging from 8 to 40 . For these analyses, we use the data provided by the veterans in 1984 (Time 1) because it is closer in time to the events. Internal consistency of the combat exposure was excellent in the current study with Cronbach's alphas of 0.93 in 1984 and 0.95 in 1998 . For comparability with our previous report, the scores were categorized as low (8-15), medium (16$25)$, or high (26-40), with 520 (38\%), 566 (41\%), and $291(21 \%)$ in each combat category, respectively.

Posttraumatic stress disorder symptoms were evaluated at both times using the 17-item 5-point Likert-type PTSD Symptom Frequency Scale (Snow et al., 1988) developed for the 1984 survey, and following the diagnostic criteria for PTSD as defined by the Diagnostic and Statistical Manual of Mental Disorders, Third Edition (DSM-III; American Psychiatric Association, 1980). Veterans were asked how frequently they experienced each symptom in the past month with response options ranging from never to very often. Because the PTSD symptoms from the DSM-III were identical to those in the Diagnostic and Statistical Manual of Mental Disorders, Third Edition, Revised (DSMIII-R; American Psychiatric Association, 1987), presumptive PTSD diagnosis was determined according to DSM$I I I-R$ criteria to make results comparable to other studies of Vietnam veterans (Yehuda \& McFarland, 1995). That is, the veteran must report often or very often to experiencing one symptom in the intrusive reexperiencing cluster, three symptoms in the avoidance/numbing cluster, and two symptoms in the arousal cluster to meet diagnostic criteria. 


\section{Statistical Analuses}

What is the reliability of combat exposure reporting over 14 years? To examine test-retest reliability, Pearson correlation coefficients were calculated for the association between total combat exposure score at Time 1 and Time 2. As in Southwick et al. (1997) and King et al. (2000), we calculated four frequency distributions to describe the extent of change in the sample: (a) changes from nonendorsement to endorsement or shifts from never to rarely or more, (b) changes from endorsement to nonendorsement or shifts from rarely or more to never, (c) total number of changes in endorsement, and (d) any changes at all. We then examined whether participants' changes in reporting altered their combat exposure category assignment, e.g., whether they moved from being assigned as low combat in 1984 and were reassigned as high combat in 1998 .

Are changes in combat exposure reports associated with changes in PTSD symptoms or presumptive PTSD diagnosis? Pearson correlations were used to test the association between PTSD symptoms at Time 2 and the number of changes in the combat exposure items over time. Next, we tested whether changes in specific PTSD symptom clusters were associated with changes in endorsement. To accomplish this, we used two ordinary least square regression models with difference scores for reexperiencing, avoidance, and arousal symptoms as the independent variables and number of items changed from never to endorsement and endorsement to never as the dependent variables.

Finally, we examined distributions of changes in reporting by PTSD status. For this purpose, participants were classified into four groups according to whether and when they met criteria for PTSD diagnosis: (a) PTSD diagnosis at Time 1 and $2(n=78)$, (b) PTSD diagnosis at Time 1 only $(n=94)$, (c) PTSD diagnosis at Time 2 only $(n=76)$, and (d) No PTSD diagnoses $(n=1214)$. We then conducted a 4 (PTSD Group) $\times 2$ (Time: 1984, 1998) analysis of variance (ANOVA). Time was a within-subjects variable, which tested whether combat reporting signifcantly changed over time. PTSD group was a betweensubjects variable, which tested whether change in exposure reporting over time differed by groups defined according to
PTSD diagnosis. Univariate repeated-measures ANOVAs were conducted to examine change over time within a PTSD group when the Wilks's lambda was significant for the PTSD Group $\times$ Time Interaction term. These analyses were conducted for the combat exposure total and each combat exposure item as dependent variables.

The effect size for each group was calculated from the mean change (difference score) in the total combat score from Time 1 to Time 2, divided by the population standard deviation for the observed change in combat exposure total. (Effect sizes: $\leq 0.20=$ small, $0.50=$ medium, and $\geq 0.80$ or more = large; Cohen, 1992). In this study, mean change was calculated to be consistent with direction, with positive change values corresponding to increased combat exposure reporting and vice versa.

What is the degree to which changes in combat exposure reports affect the dose response relation with PTSD symptoms/diagnosis? We examined the effect of recall bias on the observed dose-response relation between combat exposure reports and PTSD in two ways. First, we treated combat as a categorical variable. We then regressed Time 2 PTSD diagnosis on combat exposure (low, medium, high) evaluated at Time 1, using a binary logistic model. Next, we regressed Time 2 PTSD on categorical combat exposure evaluated at Time 2 . We then compared the odds ratios for the association between combat exposure at Time 2 and PTSD at Time 2 with the odds ratios for the association between combat exposure at Time 1 and PTSD at Time 2. This comparison gives a sense of the degree to which changes in combat exposure reports affect the observed dose-response relation between combat exposure and PTSD. We conducted a similar analysis treating combat exposure and PTSD as continuous variables.

\section{RESULTS}

At the time of the 1998 survey, the mean age was 52 years $(S D=5.38) ; 98.7 \%$ were non-Hispanic White; $75.1 \%$ lived with a spouse or partner; $37.4 \%$ had a high school education or less, $40.9 \%$ had some college, and $20.9 \%$ had a college education or more; $68.5 \%$ had a family income of over $\$ 50,000$ in 1998 . In terms of branch of service, 
63.0\% were in the Army, $10.3 \%$ the Navy, $10.7 \%$ the Air Force, $14.6 \%$ in the Marines, and $1.5 \%$ did not indicate their branch of service.

Veterans who did not respond at Time 2 were not different on any demographic variables from those who responded. However, those who did not respond in 1998 had slightly lower combat exposure $(M=13.02, S D=7.48)$ than those who responded at both times $(M=13.02, S D=7.48), t(6154)=-2.07$, $p<.05$. Those who did not respond also had higher PTSD symptoms in $1984(M=43.72, S D=13.62)$ than those who responded $(M=37.37, S D=14.54)$, $t(2906.60)=-13.69, p<.001$.

\section{Reliability of Combat Reports}

What is the reliability of combat exposure reporting over 14 years? Scores on the total CES at Time $1(M=19.18$, $S D=7.89)$ and Time $2(M=19.23, S D=8.16)$ were highly correlated, $r(1462)=.87, p<.001$, indicating excellent test-retest reliability.

Table 1 presents the frequency distributions for the type and number of combat exposure changes in the sample. We found that $59.3 \%$ of respondents did not change from never to endorsement on any item, whereas $54.9 \%$ did not change from endorsement to never on any item. Most individuals made at least one change in one combat exposure item as only $6.3 \%$ of individuals responded identically to all items at both time periods.

The distribution of respondents in combat exposure categories (low, medium, high) was highly consistent. The majority of respondents $(77.2 \%)$ did not change combat exposure category. A minority (22.4\%) made a one-step category change (e.g., from low to medium or vice versa). Only five individuals changed two categories.

\section{Combat Reports and PTSD}

Are changes in combat exposure reports associated with changes in PTSD symptoms or presumptive PTSD diagnosis? The correlations between PTSD symptoms in 1998 and the number of items changed from never to endorsement, $r(1,224)=.03$, ns or from endorsement to never were not significant, $r(1,224)=.04, n s$. A small but significant association was found between PTSD symptoms in 1998 and the number of items changed from never to endorsement or endorsement to never, $r(1,224)=-.08$,

Table 1. Number of Responses Changed per Subject on Combat Exposure Measure From 1984 to $1998(N=1462)$

\begin{tabular}{|c|c|c|c|c|c|c|c|c|}
\hline \multirow{3}{*}{$\begin{array}{l}\text { Number of responses } \\
\text { changed per participant }\end{array}$} & \multicolumn{8}{|c|}{ Type of change } \\
\hline & \multicolumn{2}{|c|}{ Never to endorsement } & \multicolumn{2}{|c|}{ Endorsement to never } & \multicolumn{2}{|c|}{ Either $^{a}$} & \multicolumn{2}{|c|}{ Any change $e^{b}$} \\
\hline & $N$ & $\%$ & $N$ & $\%$ & $N$ & $\%$ & $N$ & $\%$ \\
\hline 0 & 867 & 59.3 & 802 & 54.9 & 566 & 38.7 & 92 & 6.3 \\
\hline 1 & 249 & 17.0 & 304 & 20.8 & 333 & 22.8 & 175 & 12.0 \\
\hline 2 & 74 & 5.9 & 98 & 6.7 & 178 & 12.2 & 206 & 14.1 \\
\hline 3 & 34 & 2.7 & 39 & 2.7 & 89 & 6.1 & 193 & 13.2 \\
\hline 4 & 17 & 1.4 & 12 & 0.8 & 50 & 3.4 & 204 & 14.0 \\
\hline 5 & 12 & 1.0 & 2 & 0.1 & 29 & 2.0 & 169 & 11.6 \\
\hline 6 & 3 & 0.2 & 0 & 0.0 & 7 & 0.5 & 119 & 8.1 \\
\hline 7 & 0 & 0.0 & 0 & 0.0 & 2 & 0.1 & 66 & 4.5 \\
\hline 8 & 1 & 0.1 & 0 & 0.0 & 3 & 0.2 & 33 & 2.3 \\
\hline Missing & 205 & 14.0 & 205 & 14.0 & 205 & 14.0 & 205 & 14.0 \\
\hline
\end{tabular}

${ }^{\mathrm{a}}$ Change from never to endorsement or from endorsement to never. ${ }^{\mathrm{b}}$ Any change of any degree, e.g., from never to endorsement or from rarely to often. 
Table 2. Number of Responses Changed per Subject on Combat Exposure Measure From 1984 to 1998 by Posttraumatic Stress Disorder (PTSD) Status

\begin{tabular}{|c|c|c|c|c|c|c|c|c|}
\hline \multirow[b]{2}{*}{$\begin{array}{l}\text { Number of } \\
\text { responses } \\
\text { changed per } \\
\text { participant }\end{array}$} & \multicolumn{4}{|c|}{ Never to endorsement } & \multicolumn{4}{|c|}{ Endorsement to never } \\
\hline & $\begin{array}{c}\text { PTSD } \\
\text { Both times } \\
(n=78)\end{array}$ & $\begin{array}{c}\text { PTSD } \\
\text { Time } 1 \text { only } \\
(n=94)\end{array}$ & $\begin{array}{c}\text { PTSD } \\
\text { Time } 2 \text { only } \\
(n=76)\end{array}$ & $\begin{array}{l}\text { No PTSD } \\
(n=1214)\end{array}$ & $\begin{array}{c}\text { PTSD } \\
\text { Both times }\end{array}$ & $\begin{array}{c}\text { PTSD } \\
\text { Time } 1 \text { only }\end{array}$ & $\begin{array}{c}\text { PTSD } \\
\text { Time } 2 \text { only }\end{array}$ & No PTSD \\
\hline 0 & $67.9 \%$ & $69.1 \%$ & $53.9 \%$ & $58.3 \%$ & $60.3 \%$ & $64.9 \%$ & $59.2 \%$ & $53.5 \%$ \\
\hline 1 & 7.7 & 14.9 & 11.8 & 18.1 & 17.9 & 16.0 & 10.5 & 22.0 \\
\hline 2 & 5.1 & 2.1 & 10.5 & 4.9 & 7.7 & 4.3 & 7.9 & 6.8 \\
\hline 3 & 2.6 & 0 & 2.6 & 2.5 & 1.3 & 0 & 5.3 & 2.8 \\
\hline 4 & 1.3 & 0 & 2.6 & 1.2 & 0 & 1.1 & 0 & 0.9 \\
\hline 5 & 2.6 & 0 & 1.3 & 0.7 & 0 & 0 & 0 & 0.2 \\
\hline 6 & 0 & 0 & 0 & 0.2 & 0 & 0 & 0 & 0 \\
\hline 7 & 0 & 0 & 0 & 0 & 0 & 0 & 0 & 0 \\
\hline 8 & 0 & 0 & 0 & 0.1 & 0 & 0 & 0 & 0 \\
\hline Missing & 12.8 & 13.8 & 17.1 & 13.9 & 12.8 & 13.8 & 17.1 & 13.9 \\
\hline
\end{tabular}

$p<.01$. The association for the number of items changed at all was also significant, $r(1,224)=.30, p<.001$.

The regression results indicated that increases in reexperiencing $(B=0.02, S E=0.01, \beta=.08)$, but not avoidance $(B=0.007, S E=0.007, \beta=.03)$ and arousal $(B=0.01, S E=0.008, \beta=.05)$ symptoms were positively and significantly associated with the number of items changed from never to endorsement.

Table 2 compares the number of items changed by individuals who did not have PTSD with those who had PTSD at either or both assessments. The proportion of participants in a group who changed at least one item in the combat exposure can be calculated by subtracting the percentage in the first row from 100. Of those with PTSD at both times, $32.1 \%$ changed at least one item from never to endorsement. The largest proportion of changes to endorsement was in the PTSD Time 2 only group (46.1\%), with the smallest number in the PTSD Time 1 only group (30.9\%). The largest proportion of changes from endorsement to never was in the No PTSD group (46.5\%) and the smallest number in the PTSD Time 1 only group (35.1\%). The respective proportions for the group with PTSD at both times and the PTSD Time 2 group were 39.7\% and $40.8 \%$.
Figure 1 presents the repeated measures ANOVA results for comparison of the total CES combat score among the four PTSD diagnostic groups. The Time effect, Wilks's $\lambda=7.46, p<.01$, and the PTSD $\times$ Time Interaction, Wilks's $\lambda=15.52, p<.001$ were both significant. Simple effects analyses indicated that CES total changed significantly for the PTSD at both times $(p<.01$; effect size $=0.33)$, PTSD Time $1(p<.01$; effect size $=-0.34)$, and PTSD Time $2(p<.01$; effect size $=0.38)$ groups. The change was in the direction of a decrease for the PTSD Time 1 only group, but manifested as an increase for the other groups. CES total did not change for the No PTSD group (effect size $=-0.005$ ).

Table 3 presents the difference scores in standard deviation units and repeated measures ANOVA results for each combat item. The Time effect was significant for items 3, 4, and 6. The PTSD $\times$ Time Interaction term was significant for all items. Simple effects analysis indicated that the mean score for combat items $3(p<.001)$, $4(p<.001), 5(p<.001)$, and $7(p<.001)$ increased significantly for the group with PTSD at both times. The mean score for combat items $4(p<.05), 5(p<.05)$, $6(p<.05), 7(p<.001)$, and $8(p<.001)$ decreased significantly for the PTSD Time 1 group. The mean score 


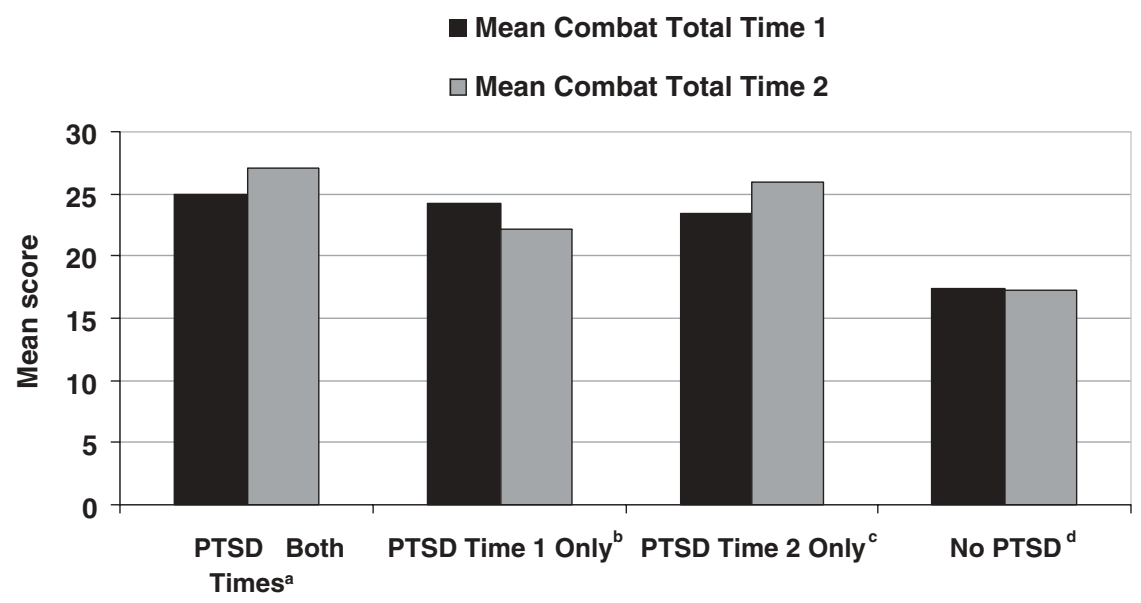

Figure 1. Mean value of total combat score at Time 1 (1984) and Time 2 (1998) by posttraumatic stress disorder (PTSD) group. ${ }^{a}$ Significant time effect $(F(1,67)=7.29, p<.01)$. ${ }^{b}$ Significant time effect $(F(1,80)=8.68, p<.01)$. ${ }^{\text {CSignificant time effect }}$ $(F(1,62)=9.18, p<.01) .{ }^{\mathrm{d}}$ No significant time effect $(F(1,1044)<1, n s)$.

for combat items $2(p<.05), 3(p<.01), 4(p<.01)$, $6(p<.001), 7(p<.05), 8(p<.01)$ increased significantly for the PTSD Time 2 group. The No PTSD group significantly decreased on item $1(p<.05)$ and increased on item $8(p<.01)$.

What is the degree to which changes in combat exposure reports affect the dose response relation with PTSD symptoms/diagnosis? The dose-response relation between combat exposure at Time 2 and PTSD diagnosis at Time 2 was nearly double that at Time 1 . Using Time 2 low combat exposure $(n=512)$ as the reference group, the association between PTSD at Time 2 for medium combat exposure $(n=531)$ was $\mathrm{OR}=5.0(95 \%$ $\mathrm{CI}=2.6-9.8)$, and for high combat exposure $(n=290)$ was $\mathrm{OR}=15.5(95 \% \mathrm{CI}=8.1-30.0)$. Using Time 1 low combat exposure $(n=520)$ as the reference group, the association between PTSD at Time 2 was for medium combat exposure $(n=566)$ OR $=3.0(95 \% \mathrm{CI}=1.8$ $5.1)$, and for high combat exposure $(n=291) \mathrm{OR}=7.0$ $(95 \% \mathrm{CI}=4.2-12.0)$. The linear regression models, where PTSD symptoms at Time 2 were regressed on combat exposure measured continuously at Time 1 , yielded $B=0.76$, $S E=0.05, \beta=.41, p<.001$. When we regressed PTSD symptoms at Time 2 on combat exposure measured con- tinuously at Time 2 we obtained $B=0.90, S E=0.04$, $B=.50, p<.001$. The differences in the regression coefficients using Time 1 versus Time 2 reports was statistically significant, $\chi^{2}(1, n=1462)=4.78, p<.05$. However, the effect size of the changes was small; the $\beta$ increased by 0.09 when Time 2 combat reports are used instead of Time 1 reports. Thus, the effect of changes in reporting on the relationship between combat exposure and PTSD are much less substantial when the continuous measures of exposures and PTSD were used.

\section{DISCUSSION}

The results of this investigation show that although combat exposure scores show excellent test-retest reliability over a 14-year period, changes in combat exposure reports occur. Furthermore, such changes are positively associated with changes in PTSD symptoms. Veterans who met criteria for a presumptive diagnosis of PTSD in 1984 but not in 1998 showed, on average, a significant decrease in combat exposure reported, and those who met criteria in 1998 but not in 1984 showed a significant increase. Although the effect sizes for these changes were small, they resulted in a stronger dose-response relationship between combat 


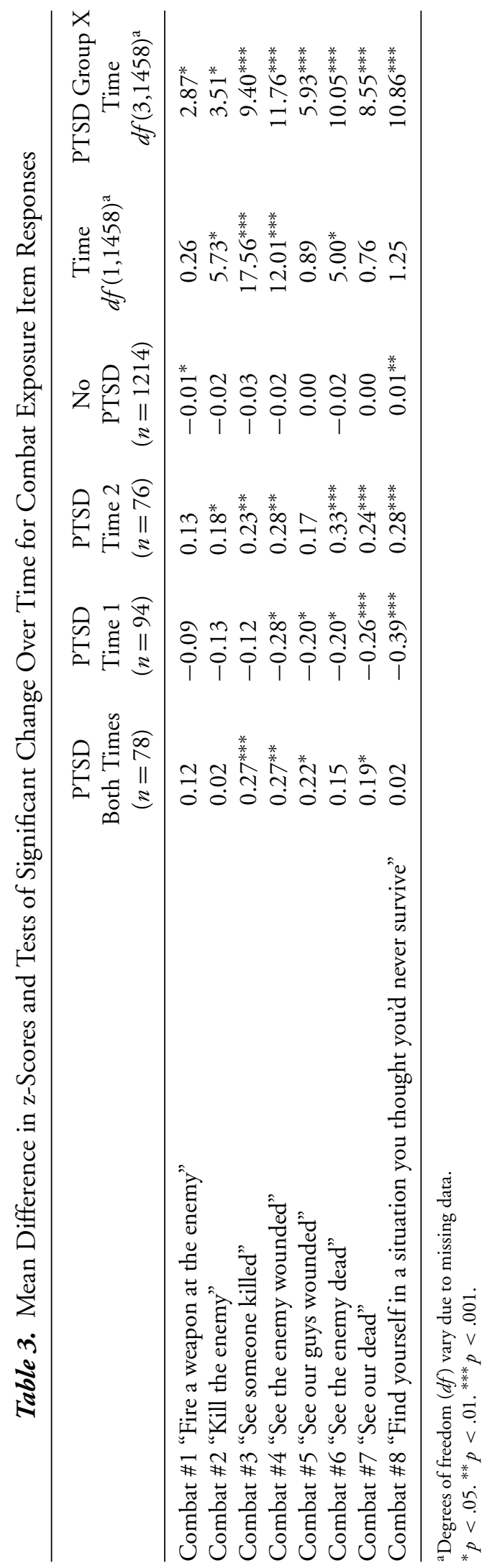

exposure and PTSD when both were assessed at Time 2. This inflation was particularly marked when combat exposure and PTSD were treated as categorical variables. Such models are inherently less precise than the continuous variable model. Finally, increases in exposure reports were specifically predicted by increases in reexperiencing symptoms. This suggests the presence of intrusive memories of the Vietnam combat experience may drive veterans' reports of exposure.

The pattern of a positive association between changes in PTSD status and changes in combat exposure reporting continued when changes in endorsement and frequency of individual combat items were examined. A higher proportion of those who met the criteria for PTSD in 1998, but not in 1984, changed their responses to at least one item from never to endorsement, than those with PTSD at both periods or those with PTSD in 1984 only. Following this pattern, veterans who had PTSD in 1984, but not in 1998 showed significant decreases in the mean responses on five items and those with PTSD in 1998, but not 1984 showed significant increases on six items.

In our study, combat exposure reporting was more consistent (test-retest correlation of .87) than that observed by Roemer et al. (1998; test-retest correlation of .66) and King et al. (2000; test-retest correlation of .61). Moreover, a smaller proportion of our participants showed changes in their endorsement of items, $61.3 \%$, than that found previously (e.g., 91.1\% in King et al., 2000 and 88.0\% in Southwick et al., 1997). Changes in reporting were more often in terms of frequency (e.g., from rarely to often) than in endorsement. The degree of change in reporting on individual items was also relatively small with changes ranging from .01 to .39 standard deviations. Finally, the great majority of veterans $(77.2 \%)$ did not change their combat exposure category (low, medium, or high) over the 14-year period.

The previous studies with lower reliability took initial combat exposure assessments within 5 days to one-year following the participants' return to the United States and participants were not Vietnam veterans. Our survey was not conducted until 1984, 10 years or more after our participants returned from Southeast Asia, which was also the 
case with Janes et al. (1991), who studied Vietnam veterans and found a high test-retest reliability. It is possible that the largest changes in exposure reporting occur within the first few years of initial exposure when PTSD symptoms are purported to be especially acute. After that time, few changes in recall of exposure may occur, although the different directions of change observed in Time 1 only and Time 2 only groups do not support this argument. Our participants' exposure reports might also have been more consistent due to differences in the nature of the Vietnam experience when compared to that of the Gulf War or Somali peacekeeping experience assessed in previous investigations. Unfortunately, neither objective measures of the actual combat experienced nor baseline measures of combat exposure soon after the exposure occurred are available for our sample. Janes et al. (1991), however, did validate their responses by comparison with whether or not the respondent received a combat-related war medal.

Finally, certain psychometric qualities of our CES scale might also have contributed to its greater stability over that used by others (King et al., 2000; Southwick et al., 1997). Scales are designed to provide information on an underlying or latent construct that cannot be observed directly. Scales with multiple items in a Likert-type response format are more reliable than single items or those using a binary response format. Our findings also demonstrate the great difficulty in analyzing individual items within a scale.

Because our data do not provide information on the accuracy of participants' memories of their combat experiences, we cannot know whether these changes produced inflated or more accurate reports. Changes in reporting were positively associated with changes in PTSD symptoms and thus our data suggest that the very symptoms of PTSD most related to the veteran's memory of his combat experiences' reexperiencing symptoms, are most related to increases in exposure reporting. This suggests participants with PTSD had these experiences more readily available to recall when they were filling out the checklist (Tversky \& Kahneman, 1973). Furthermore, a large body of literature has documented that the search for meaning is an important part of recovery from traumatic experiences (Herman, 1992 Janoff-Bulman, 1992). One aspect of meaning making involves constructing a coherent narrative of traumatic events within the context of the rest of one's life. It is possible that changes in reporting by our participants with PTSD are related to this search for meaning. Another possible explanation for the association between changes in exposure reporting and changes in PTSD symptoms can be found in the literature on mood dependent recall (Cohen et al., 1988). For example, major depression, a condition commonly comorbid with combat-related PTSD (Kulka et al., 1990) is associated with systematic biases in the processing of information that lead to high-frequency estimates of negative events. Another possibility is that although part of the increase at Time 2 is due to experiencing symptoms among those with PTSD, the decrease in exposure reports among those with PTSD at Time 1 may only be because those veterans have recovered from the disorder and have put their Vietnam experiences behind them.

Because the combat exposure reporting over a 14-year period was highly consistent for the overall sample, it appears that the combat exposure measure is a useful instrument for epidemiological studies involving veterans' combat experiences. Changes in exposure reporting, when they occur, are associated with changes in PTSD symptoms and these findings, together with those of previous studies call into question the unidirectional dose-response relationship between exposure severity and PTSD that has been important for the validation of the syndrome. Poor recall per se, which we do not observe, would lead to random error in exposure reports and reduced power to test hypotheses. The small but significant changes in recall observed here are systematically biased in favor of the alternative hypothesis (e.g., that risk of developing PTSD increases with exposure severity) are problematic and can lead to distorted dose-response relationships in the type of cross-sectional research most commonly done in the trauma field.

Our findings have several practical implications for researchers in the field of trauma and PTSD. First, the correlation of changes in PTSD symptoms with changes in exposure reports over time highlights the need for longitudinal studies of trauma survivors. Issues of recall bias are not absent from such studies. However, longitudinal 
studies have the ability to disentangle exposure and symptom reports as well as to examine within-individual change over time. For example, using data from the same sample as the present study, we examined the association between combat exposure severity assessed at Time 1 and change in PTSD over the 14-year follow-up and found that combat exposure was the strongest predictor of PTSD course in this sample (Koenen et al., 2003). Second, to improve recall generally, combat assessment in military populations, especially those returning from war theaters, should be done as close in time to the events as possible. Such assessments would also provide a baseline for longitudinal follow-ups. Third, future research might benefit from combining selfreports of exposure with independent sources of data such as military records, contemporary journalists' accounts, and other documents (e.g., Dohrenwend et al., 2006). Researchers studying the mental health consequences of civilian traumas such as motor vehicle accidents and natural disasters are increasingly employing independent assessments of trauma severity. These assessments include physician's ratings of medical injury severity and reports by key informants (e.g., parents, spouses; see Hall et al., 2006; Saxe et al., 2005). Such other sources have their own inadequacies and biases. However, their biases are likely to be independent of participants' reports of symptomatology. Ultimately, the trauma field would be advanced if better record keeping and quantitative objective measures of combat trauma were available.

\section{REFERENCES}

American Psychiatric Association. (1980). Diagnostic and statistical manual of mental disorders (3rd ed.). Washington, DC: Author.

American Psychiatric Association. (1987). Diagnostic and statistical manual of mental disorders (3rd ed., rev.). Washington, DC: Author.

Cohen, J. (1992). A power primer. Psychological Bulletin, 112, 155159.

Cohen, L. H., Towbes, L. C., \& Flocco, R. (1988). Effects of induced mood on self report life events and perceived and received social support. Journal of Personality and Social Psychology, 55, 669674 .
Dohrenwend, B. P., Turner, J. B., Turse, N. A., Adams, B. G., Koenen, K. C., \& Marshall, R. (2006, August 18). Controversy over the psychologic risks of Vietnam for U.S. veterans: A revisit with new data and methods. Science, 313, 979-982.

Figley, C. R. (1978). Stress disorders among Vietnam veterans. New York: Brunner/Mazel.

Frye, J. S., \& Stockton, R. (1982). Discriminant analyses of posttraumatic stress disorder among a group of Vietnam veterans. American Journal of Psychiatry, 139, 52-56.

Hall, E., Saxe, G., Stoddard, F., Lopez, C., Kaplow, J., Koenen, K. C., et al. (2006). Posttraumatic stress symptoms in parents of children with acute burns. Journal of Pediatric Psychology, 31, 403-412.

Herman, J. (1992). Trauma and recovery. New York: Basic Books.

Janes, G. R., Goldberg, J., Eisen, S. A., True, W. R., \& Henderson, W. G. (1991). Reliability and validity of a combat exposure index for Vietnam era veterans. Journal of Clinical Psychology, 47, 8086.

Janoff-Bulman, R. (1992). Shattered assumptions: Towards a new psychology of trauma. New York: The Free Press.

King, D. W., \& King, L. A. (1991). Validity issues in research on Vietnam veteran adjustment. Psychological Bulletin, 109, 107124.

King, D. W., King, L. A., Erickson, D. J., Huang, M. T., Sharkansky, E. J., \& Wolfe, J. (2000). Posttraumatic stress disorder and retrospectively reported stressor exposure: A longitudinal prediction model. Journal of Abnormal Psychology, 109, 624-633.

Koenen, K. C., Stellman, J. M., Stellman, S. D., \& Sommer, J. F., Jr. (2003). Risk factors for course of PTSD among Vietnam veterans: A 14-year follow-up of American Legionnaires. Journal of Consulting \& Clinical Psychology, 6, 980-986.

Kulka, R. A., Schlenger, W. E., Fairbank, J. A., Hough, R. L., Jordan, B. K., Marmar, C. R., et al. (1990). Trauma and the Vietnam War generation. New York: Brunner/ Mazel.

Roemer, L., Litz, B., Orsillo, S., Ehlich, P. J., \& Friedman, M. J. (1998). Increases in retrospective accounts of war-zone exposure over time: The rise of PTSD symptom severity. Journal of Traumatic Stress, 11, 597-605.

Saxe, G., Miller, A., Bartholomew, D., Hall, E., Lopez, C., Kaplow, J. B., et al. (2005). Incidence of and risk factors for acute stress disorder in children with injuries. Journal of Trauma, Injury, Infection, and Critical Care, 59, 946-953.

Snow, B. R., Stellman, J. M., Stellman, S. D., \& Sommer, J. F., Jr. (1988). Posttraumatic stress disorder among American Legionnaires in relation to combat experience in Vietnam: Associated and contributing factors. Environmental Research, 47, 175-192. 
Southwick, S. M., Morgan, C. A., III, Nicolau, A. L., \& Charney, D. S. (1997). Consistency of memory for combat-related traumatic events in Veterans of Operation Desert Storm. American Journal of Psychiatry, 154, 173-177.

Stellman, J. M., Stellman, S. D., \& Sommer, J. F., Jr. (1988a). Social and behavioral consequences of combat and herbicide exposure in Vietnam among American Legionnaires. Environmental Research, 47, 129-149.

Stellman, J. M., Stellman, S. D., \& Sommer, J. F., Jr. (1988b). Utilization, attitudes, and experiences of Vietnam era veterans with Veterans Administration health facilities: The American Legion experience. Environmental Research, 47, 193-209.

Stellman, S. D., Stellman, J. M., \& Sommer, J. F., Jr. (1988a). Combat and herbicide exposure in Vietnam among a sample of American Legionnaires. Environmental Research, 47, 112128.

Stellman, S. D., Stellman, J. M., \& Sommer, J. F., Jr. (1988b). Health and reproductive outcomes among American Legionnaires in relation to combat and herbicide exposure in Vietnam. Environmental Research, 47, 150174.

Tversky, A., \& Kahneman, D. (1973). Availability: A heuristic for judging frequency and probability. Cognitive Psychology, 5, 207232.

Yehuda, R., \& McFarlane, A. C. (1995). Conflict between current knowledge about posttraumatic stress disorder and its original conceptual basis. American Journal of Psychiatry, 152, 17051713. 